Cite this: Phys Chem Chem. Phys. 2014, 16, 17081

Received 28th March 2014 Accepted 1st July 2014

DOI: $10.1039 / c 4 c p 01369 g$

www.rsc.org/pccp

\section{Electrochemical reductive desorption of alkyl self-assembled monolayers studied in situ by spectroscopic ellipsometry: evidence for formation of a low refractive index region after desorption $\dagger$}

\begin{abstract}
Nina Kemnade, $\$$ Ying Chen, $\S$ Mutlu I. Muglali and Andreas Erbe*
Gold-sulphur bonds holding self-assembled monolayers (SAMs) on their gold substrate can be broken by electrochemical reduction, which typically occurs in an electrode potential range where the electrochemical hydrogen evolution reaction (HER) is thermodynamically possible. This work uses an in situ coupling between cyclic voltammetry and spectroscopic ellipsometry to compare the interfacial structure after desorption of the aliphatic thiols 1-Dodecanethiol (DDT) and 1-Octadecanethiol (ODT), and the $\omega$-hydroxythiol 11-Mercapto-1-undecanol (MUD). For MUD and DDT, the data can only be explained by the presence of a substance with a significantly lower refractive index than the aqueous electrolyte in the interfacial region. This substance is likely to be $\mathrm{H}_{2}$. The hypothesis is put forward here that for MUD and DDT, desorbed molecules stabilise "nanobubbles" of $\mathrm{H}_{2}$. The resulting aggregates form as initial stages of the process of complete disintegration of the SAMs, i.e. the loss of the SAMforming molecules into solution. On the other hand, desorption and readsorption of ODT are fully reversible the presence of a layer with low refractive index can neither be excluded nor confirmed in this case. The results indicate that different SAM-stabilities are a consequence of solubility of the thiolates.
\end{abstract}

\section{Introduction}

In recent years, the existence of bubbles in the size range of tens of $\mathrm{nm}$ at solid/liquid interfaces of hydrophobic solids has been debated..$^{1-3}$ Evidence for the existence of such "nanobubbles" has been found e.g. on thiol-modified and pure gold by quartz crystal microbalance measurements, ${ }^{4,5}$ or by scanning probe microscopy on graphite, ${ }^{6,7}$ in contact with water or aqueous solutions. On the other hand, ellipsometric measurements on hydrophobic silane-modified silicon samples have yielded no hints about the existence of gases at the solid/water interface, ${ }^{8}$ though from the point of view of hydrophobicity, these samples

Max-Planck-Institut für Eisenforschung GmbH, Max-Planck-Str. 1, 40237 Düsseldorf, Germany.E-mail: a.erbe@mpie.de, aerbe@arcor.de; Fax: +49211 6792218; Tel: +492116792890

$\dagger$ Electronic supplementary information (ESI) available: Desorption curves of MUD, DDT and ODT in $\mathrm{NaClO}_{4}$. Apparent wavelength dependence of apparent hydrogen layer thickness. See DOI: 10.1039/c4cp01369g

‡ Present address: Institute of Physical Chemistry, Westfälische WilhelmsUniversität Münster, Corrensstraße 28/30, 48149 Münster, Germany.

$\S$ Present address: Physik-Department E19, Technische Universität München, James-Franck-Straße 1, 85748 Garching, Germany.

ๆ Present address: Atotech Deutschland GmbH, Erasmusstraße, 10553 Berlin. should be similar to those of thiol-modified gold used in a later study. ${ }^{4}$ In an electrochemical gas evolution reaction, such as the hydrogen evolution reaction (HER) in aqueous solutions, $\mathrm{H}_{2} \mathrm{O}+\mathrm{e}^{-} \rightarrow 1 / 2 \mathrm{H}_{2}+\mathrm{OH}^{-}$or $\mathrm{H}^{+}+\mathrm{e}^{-} \rightarrow 1 / 2 \mathrm{H}_{2}$, gas bubbles do form and they could be of nano-dimension in the initial stages. An electrochemical experiment enabling a controlled gas generation at the interface should therefore be well-suited to study the formation of the mentioned nanobubbles, and their effect on interfacial properties. To ensure the presence of a hydrophobic surface, metals can be modified by self-assembled monolayers (SAMs), in which organic molecules are linked via a sulphur atom to a metal, such as gold. ${ }^{9-14}$

These SAMs on the other hand undergo a reductive desorption process, $\|$ in which the $\mathrm{Au}-\mathrm{S}$ bond is broken. ${ }^{15}$ Reductive desorption usually occurs in an electrode potential range in which HER is thermodynamically possible. ${ }^{16}$ However, HER is typically observed only after the reductive desorption. ${ }^{15}$ The result is a potentially complex interplay between the two

|| In accordance with the electrochemical literature, the term "desorption" in this work will be used to refer to the break of the Au-S bond ("chemi-desorption"). After break of the Au-S bond, molecules may stay physisorbed to the solid electrode and/or slowly move away from the interface ("physi-desorption"). 
reactions, where the organic molecules in the interfacial region affect the gas evolution, and hence bubble formation nucleation. A deeper study of this interplay shall serve as the main motivation for this manuscript.

Reductive desorption limits the SAM's application range, as it defines a fundamental stability limit. Because SAM stability is crucial for applications, ${ }^{17}$ it is essential to understand SAM degradation mechanisms in electrolytes for use of SAMs in electrolytic media. SAMs act as barriers separating water and ions from the metal surface, ${ }^{18}$ which have been used e.g. to protect surfaces. ${ }^{19-23}$ Nevertheless, ions penetrate through SAMs, which is likely to happen at defects in the SAM structure. ${ }^{24}$ Controlling defects hence leads to greater SAM stability. ${ }^{25}$ Ions that penetrated the SAM and reach the metal surface affect reductive desorption, ${ }^{24,26}$ as does surface crystal structure. ${ }^{27}$ Here, we argue that the reductive desorption of thiols can be used as a model for one particular possible mechanism of SAM disintegration.

Reductive monolayer desorption results in a cathodic current peak in voltammetric analyses, however, in numerous studies, additional peaks have been observed in voltammograms. Based on in situ spectroscopic and microscopic studies, these additional peaks have mostly been related to transformations in the molecular arrangement after desorption. ${ }^{28-30}$ For desorbed long-chain alkanethiolates, the formation of aggregates which remain near the interface is generally accepted. ${ }^{31-34}$ Molecular order and orientation of the molecules within such aggregates and before desorption at the electrified interface has recently been studied extensively by vibrational sum-frequency-generation (SFG) spectroscopy, ${ }^{35-39}$ and ex situ after treatment at different potentials. ${ }^{18}$

$\mathrm{H}_{2}$ formation and the resulting drag has recently been shown to be decisive for the transport of desorbed fluorescently-labelled SAM-forming molecules into the electrolyte. ${ }^{40}$ On the other hand, the araliphatic thiol (4-(4-(4-pyridyl)phenyl)phenyl)methanethiol (PyPP1), which shows extraordinary electrochemical stability, ${ }^{41}$ has been shown to remain suspended in the interface plane after desorption, and to temporarily catalyse HER, with ellipsometric results pointing to the presence of $\mathrm{H}_{2}$ in the interfacial region after desorption. ${ }^{42}$ Similar to PyPP1, long chain alkyl thiol SAMs have also been shown to be stable over many desorption-readsorption cycles. ${ }^{18}$ In the desorbed state, ions stabilise the molecular aggregates formed. ${ }^{30}$

This work uses spectroscopic ellipsometry coupled with electrochemical experiments to investigate the state of SAMs before and after reductive desorption. As ellipsometric experiments are sensitive to interfacial refractive index, ${ }^{43}$ there is also the possibility to study the role of the evolving gas in the desorption process. Two alkyl thiols, 1-Dodecanethiol (DDT) and 1-Octadecanethiol (ODT), are used to compare the ellipsometric results with recent studies. ${ }^{18,30}$ To study the role of hydrophobicity and hydrophilicity, 11-Mercapto-1-undecanol (MUD) is the third compound in this series. Desorption is investigated in two different electrolytes: $0.1 \mathrm{M} \mathrm{NaOH}$, an electrolyte frequently used for such desorption studies, and $0.1 \mathrm{M}$ $\mathrm{NaClO}_{4}$. Because of the lower $\mathrm{pH}$ of the latter, the equilibrium potential of $\mathrm{H}_{2}$ is shifted to less negative potentials. Therefore, the rate of HER at the desorption potential is supposed to be different in the two different electrolytes.

\section{Experimental section}

\subsection{Sample preparation}

$\mathrm{Au}(111)$ on mica was used as a working electrode. For preparation of ultraflat Au films, mica sheets (Grade V1, $2.5 \mathrm{~cm} \times 7.5 \mathrm{~cm}$; Ted Pella, Inc.; USA) were freshly cleaved. On the freshly cleaved surface, gold was evaporated by electron beam evaporation in a Leybold Univex 450 chamber. Gold pellets with purity $99.999 \%$ (Wieland Edelmetalle, Pforzheim, Germany) were used. Before the evaporation, the mica sheets were heated for more than $24 \mathrm{~h}$ at $500{ }^{\circ} \mathrm{C}$ in a homemade heater to improve the adhesion of the gold film. During evaporation the temperature was kept at $500{ }^{\circ} \mathrm{C}$ and the pressure was around $8 \times 10^{-6} \mathrm{mbar}$. The gold was evaporated at an evaporation rate of $15 \mathrm{~nm} \mathrm{~min}^{-1}$. Typically a layer thickness of $150 \mathrm{~nm}$ was obtained.

The gold samples were washed with ethanol, dried in nitrogen and annealed in a hydrogen flame for 1-2 min. Afterwards they were directly immersed in a $20 \mu \mathrm{M}$ thiol solution for $12 \mathrm{~h}$ at room temperature to obtain a highly ordered monolayer. Subsequently, the samples were removed from the thiol solution, washed with ethanol and dried in a nitrogen stream.

1-Dodecanethiol (98\%, Merck), 11-Mercapto-1-undecanol (97\%, Sigma-Aldrich) and 1-Octadecanethiol (97.5\%, Fluka) were used as received from the different suppliers.

\subsection{Cyclic voltammetry (CV)}

Pure electrochemical measurements were recorded in a conventional electrochemical cell with a Compactstat potentiostat (Ivium Technologies, Eindhoven, The Netherlands). An Ag/AgCl/ $3 \mathrm{M}$ KCl-electrode (Metrohm, Filderstadt, Germany) was used as a reference electrode. A gold foil was employed as a counter electrode. The electrolyte was purged with nitrogen prior to measurement, while during the measurements, only the atmosphere above the electrolyte was purged.

All electrode potentials reported in this work are given with reference to $\mathrm{Ag} / \mathrm{AgCl} / 3 \mathrm{M} \mathrm{KCl}$.

\subsection{Ellipsometry}

A homemade electrochemical cell and a SE 800 spectroscopic ellipsometer (Sentech Instruments, Krailling/Berlin, Germany) were used for coupled ellipsometric and electrochemical experiments. Details of the cell design have been reported elsewhere. ${ }^{44,45}$ A freshly prepared sample was fixed in the cell, and the setup was aligned in the beam. The sample was connected as a working electrode to the Ivium Compactstat potentiostat. The counter electrode was a Pt-grid. Reference electrode was an Ag/AgCl-microelectrode (3 M KCl, DRIREF2SH, World Precision Instruments, Sarasota, FL, USA). The electrolyte was externally purged with nitrogen, and pumped into the cell using a peristaltic pump (Ismatec IDEX Health \& Science, Glattbrugg, Switzerland). Prior to measurements the electrolyte was pumped for $c a .10 \mathrm{~min}$ with $2 \mathrm{~mL} \mathrm{~min}^{-1}$ through 
the cell to remove oxygen. During the measurement the pump rate was reduced to $150 \mu \mathrm{L} \mathrm{min}{ }^{-1}$. Pumping showed no detectable influence on the ellipsometic data, but limited the supply of oxygen, which diffused into the cell through O-rings, and other parts. The first five ellipsometric measurements were conducted at the open circuit potential (OCP). Subsequently, a CV scan with a scan speed of $5 \mathrm{mV} \mathrm{s}^{-1}$ was started, with a potential sweep in the cathodic direction first. Typically three complete cycles were recorded in the scan range -0.2 to $-1.5 \mathrm{~V}$, unless noted otherwise. Unless noted otherwise, focus in the discussion here is on the first scan. Ellipsometric measurements were carried out during the CV scans, with a minimal protocol to reduce measurement time. Measurements were conducted using a fixed polariser, without retarder, and with the minimum of 8 analyser positions measured for one dataset. The duration of such a single ellipsometric measurement is typically $8 \mathrm{~s}$.

The ellipsometric parameters $(\Delta$ und $\Psi$ ) were measured and used to determine the ellipsometric ratio $\rho$,

$$
\rho=\frac{r_{\mathrm{p}}}{r_{\mathrm{s}}}=\tan (\Psi) \mathrm{e}^{i \Delta},
$$

with the amplitude reflection coefficients $r_{\mathrm{p}}$ and $r_{\mathrm{s}}$ for $\mathrm{p}$ - and s-polarisation, respectively, and $i=\sqrt{-1}$. Data analysis was preformed based on a perturbation approach as described by Lekner, ${ }^{46}$ and similar to the approach discussed previously. ${ }^{42,45}$ This approach is valid for layers with a thickness $d$ small compared to the vacuum wavelength $\lambda$ of the light employed. For SAMs on gold, $d \sim 1.5 \mathrm{~nm}$, while $300 \mathrm{~nm}<\lambda<820 \mathrm{~nm}$, i.e. for the topic under investigation here this approximation is sufficient. The deviation of $\rho$ from the situation in an ideal ambient-substrate model without any layer or transition region, which has the ellipsometic ratio $\rho_{0}$, can be described in a firstorder perturbation approach as ${ }^{46}$

$$
\rho=\rho_{0}-F \cdot J_{1} .
$$

The factor $F$ is comprised of optical parameters of the system, as detailed elsewhere. ${ }^{45,46}$

The perturbation parameter $J_{1}$ describes the change of the dielectric function across the interfacial region $\varepsilon_{\mathrm{s}}(z),{ }^{46}$

$$
J_{1}=\int_{-\infty}^{+\infty} \frac{\left[\varepsilon_{1}-\varepsilon_{\mathrm{s}}(z)\right]\left[\varepsilon_{\mathrm{s}}(z)-\varepsilon_{2}\right]}{\varepsilon_{\mathrm{s}}(z)} \mathrm{d} z,
$$

where index 1 indicates the incidence medium (electrolyte) and index 2 the exit medium (gold substrate). $J_{1}$ can be understood as a dielectric contrast weighted thickness, as it has the unit of a length. For the wavelength-dependence of the dielectric function $\varepsilon_{2}$ of the gold substrate values based on a critical point model have been used. ${ }^{47,48}$ The wavelength-dependence of the dielectic function $\varepsilon_{1}$ of the electrolyte was based on the wavelength-dependence of the refractive index of water, with a correction for the concentration dependence $\mathrm{d} n / \mathrm{d} c$ at a single wavelength of the electrolyte $0.1 \mathrm{M} \mathrm{NaOH} .{ }^{49}$ Small differences in the dielectric function of the electrolyte leave the results almost unaffected. Therefore, the same dielectric functions are used for both $0.1 \mathrm{M} \mathrm{NaClO}_{4}$ and $0.1 \mathrm{M} \mathrm{NaOH}$.
For a homogeneous isotropic layer between the ambient and the substrate, eqn (3) simplifies to ${ }^{46}$

$$
J_{1}=\frac{\left(\varepsilon_{1}-\varepsilon_{\mathrm{s}}\right)\left(\varepsilon_{\mathrm{s}}-\varepsilon_{2}\right)}{\varepsilon_{\mathrm{s}}} d=\left(\varepsilon_{1}+\varepsilon_{2}-\frac{\varepsilon_{1} \varepsilon_{2}}{\varepsilon_{\mathrm{s}}}-\varepsilon_{\mathrm{s}}\right) d .
$$

The authors will start the analysis assuming an isotropic layer in first approximation, and then in the course of the manuscript develop the analysis beyond isotropy.

For an experimental determination of $J_{1}$, a measurement of $\rho_{0}$ is crucial. In this work, due to experimental constraints in the repeatability of the alignment of the substrates, no repeatable measurements of $\rho_{0}$ could be performed. Therefore, the difference $\delta J_{1}$

$$
\delta J_{1}=J_{1}(E)-J_{1}(\mathrm{OCP})
$$

between measurements at electrode potential $E$ and OCP is analysed. This difference does not depend on $\rho_{0}$. Five measurements at OCP were averaged to obtain $\rho$ (OCP). Using eqn (2), the difference between the respective $\rho$ is straightforwardly related to $\delta J_{1}$ as

$$
\rho(\mathrm{OCP})-\rho(E)=F \cdot \delta J_{1}
$$

The resulting $\delta J_{1}$ are complex. Here, only $\operatorname{Im}\left(\varepsilon_{2}\right) \neq 0$. Consequently, the information content, especially about the interfacial dielectric constant, in $\operatorname{Re}\left(J_{1}\right)$ is higher than in $\operatorname{Im}\left(J_{1}\right)$. Therefore, $\operatorname{Re}\left(J_{1}\right)$ is analysed in this work.

While a direct experimental determination of values for $J_{1}$ was difficult, the alkyl SAMs analysed in this work are well characterised. Based on thickness ${ }^{50,51}$ and a Cauchy description of the dielectric function, ${ }^{52}$

$$
\varepsilon_{\mathrm{s}}(\lambda)=\left(n_{0}+\frac{n_{1}}{\lambda^{2}}\right)^{2}
$$

with parameters $n_{0}$ and $n_{1}$ as determined from ellipsometric measurements in air, the initial value $J_{1}^{(\text {init) }}$ of $J_{1}$ before any electrode potential application was determined using eqn (4). The calculated $J_{1}^{(\mathrm{init})}$ was then used to replace $J_{1}(\mathrm{OCP})$ in eqn (5), and to calculate $J_{1}(E)$.

In the examples shown in this work, two wavelengths were selected to display typical desorption curves. Values at $350 \mathrm{~nm}$ are shown, as $350 \mathrm{~nm}$ is close to a minimum in $\operatorname{Re}\left(\varepsilon_{2}\right),{ }^{47,53}$ and a wavelength where high total irradiance is recorded at the detector, and hence a good signal/noise ratio is achieved. As a sample wavelength in the mid-visible, $600 \mathrm{~nm}$ was chosen. For quantitative analysis, however, all wavelengths were considered, and resulting spectra are displayed in the ESI. $\dagger$

\section{Results and discussion}

\subsection{Bare gold electrode}

The electrochemical and ellipsometric results of the first cycle in $0.1 \mathrm{M} \mathrm{NaOH}$ of a sample of bare gold are shown in Fig. 1. In the CV of Fig. 1a, low currents in the $\mu \mathrm{A}$-range are measured till $E \sim-1.25 \mathrm{~V}$. At more negative potentials, the hydrogen evolution reaction (HER) leads to rising current densities. 


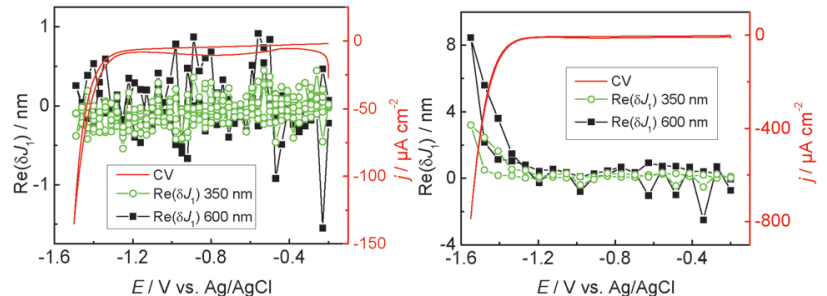

(a)

(b)

Fig. 1 First CV scan $\left(-, 5 \mathrm{mV} \mathrm{s}^{-1}\right)$ and concurrent $\operatorname{Re}\left(\delta J_{1}\right)$ at $350 \mathrm{~nm}(0)$ and $600 \mathrm{~nm}$ ( $)$ on bare $\mathrm{Au}(111)$ in $0.1 \mathrm{M} \mathrm{NaOH}$. (a) Vertex potential in cathodic scan $-1.5 \mathrm{~V}$. (b) Vertex potential in cathodic scan $-1.55 \mathrm{~V}$.

The ellipsometric data show no significant variation of $\operatorname{Re}\left(\delta J_{1}\right)$ over the full cycle. Consequently, neither the layer thickness nor the dielectric constant change significantly. The slight slope in the results for $\operatorname{Re}\left(\delta J_{1}\right)$ is presumably due to changes in the ion distribution near the electrode surface, but is on the order of the noise level observed here. Qualitatively similar results, though with a shift in the onset potential for the HER, were obtained in $0.1 \mathrm{M} \mathrm{NaClO}_{4}$.

This result shows that the measurement of ellipsometric data is possible despite a starting gas evolution reaction. When scanning to $-1.55 \mathrm{~V}$ in this example (Fig. 1b), $\operatorname{Re}\left(\delta J_{1}\right)$ is constant at $0 \mathrm{~nm}$ even after the onset of the HER. If the current increases above a certain value $\left(\approx 200 \mu \mathrm{A} \mathrm{cm}{ }^{-2}\right), \operatorname{Re}\left(\delta J_{1}\right)$ rises sharply. According to eqn (4) and (5), the increase in $\operatorname{Re}\left(J_{1}\right)$ observed there indicates the formation of a layer of a lower dielectric constant than the aqueous medium in the interfacial region. Thus, the result is consistent with the presence of gaseous $\mathrm{H}_{2}$ in the interfacial region, which is expected after the start of the HER. (The exact onset potential of the HER varies over several $10 \mathrm{mV}$ from experiment to experiment, probably because of different surface catalytic activities.) The presence of macroscopically visible bubbles on the surface leads to a decrease in overall intensity at the detector, as the bubbles act as lenses and refract light away from the ellipsometer's detector arm. Consequently, measurements are practically impossible if the electrode is completely covered with macroscopic gas bubbles.

\subsection{Desorption behaviour of MUD, DDT and ODT}

Representative results of measurements of reductive MUD desorption in $0.1 \mathrm{M} \mathrm{NaOH}$ are shown in Fig. 2. Corresponding data for DDT are shown in Fig. 3, and for ODT in Fig. 4.

The CVs of all SAMs show the well-described desorption peak originating from the break of the $\mathrm{Au}-\mathrm{S}$ bond, ${ }^{30}$ which coincides with a strong change in the ellipsometric signal. (The fine structure of these peaks has been discussed in detail in terms of the local order of the SAM, ${ }^{54,55}$ and is not a topic of this work.) In the case of DDT, there is successive cathodic multiwave desorption between $\approx-1.25 \mathrm{~V}$ and $-1.45 \mathrm{~V}$, which is superimposed to the beginning HER. These peaks do not coincide with a change in the ellipsometric signal. Therefore, these peaks are not related to a change in the layer of adsorbed
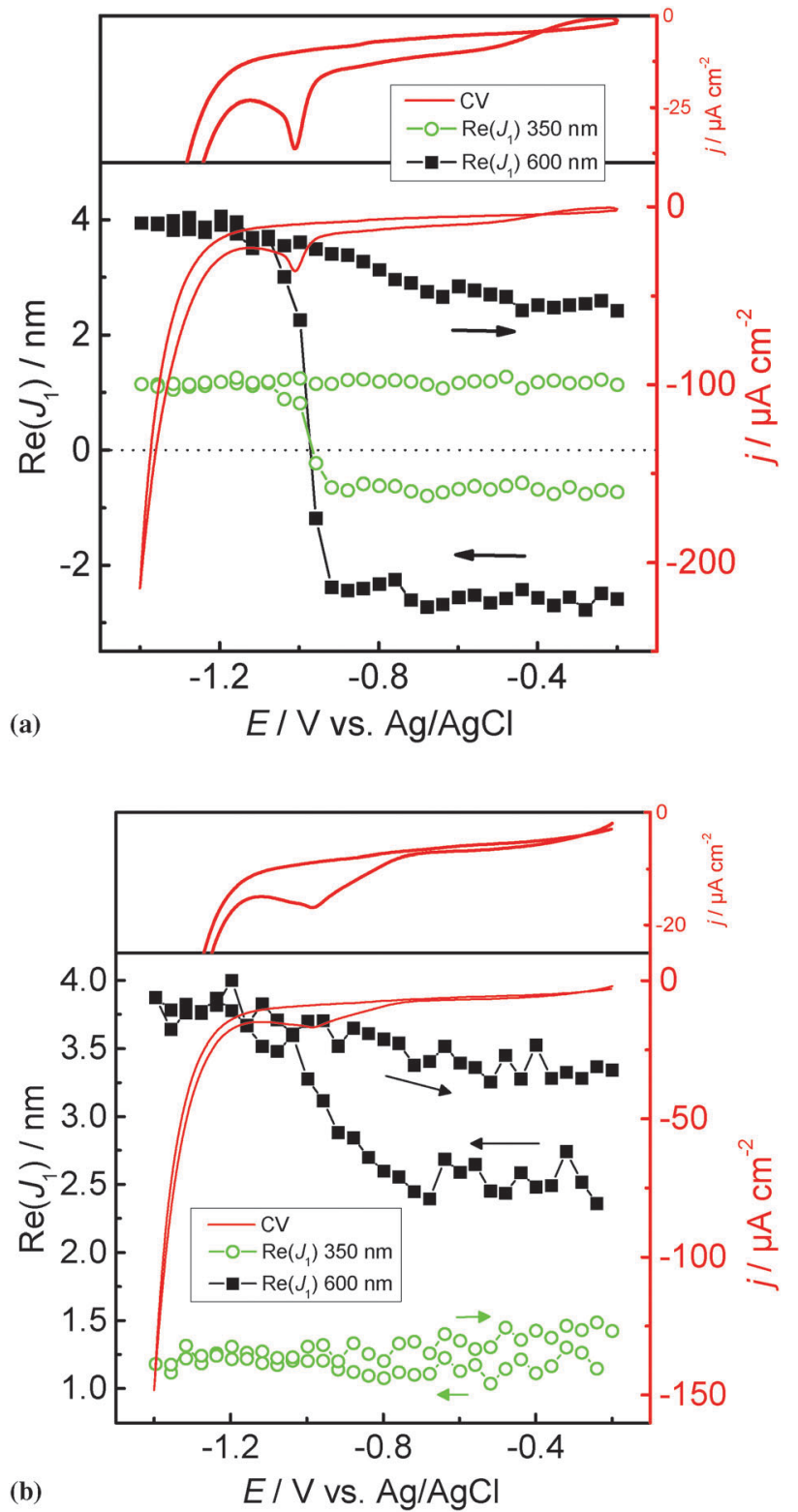

Fig. 2 (a) First and (b) second $\mathrm{CV}$ cycle $\left(-, 5 \mathrm{mV} \mathrm{s}^{-1}\right)$ and concurrent $\operatorname{Re}\left(J_{1}\right)$ at $350 \mathrm{~nm}(0)$ and $600 \mathrm{~nm}(\mathbf{\square})$ of MUD-covered $\mathrm{Au}(111)$ in $0.1 \mathrm{M}$ $\mathrm{NaOH}$. Arrows indicate the scan direction for the ellipsometric curves. The dotted line $(\cdots)$ shows $\operatorname{Re}\left(J_{1}\right)=0$.

molecules or gas, but to changes in the reactivity or structure of the surface.

In the negative scan during the first $\mathrm{CV}$ cycle in $\mathrm{NaOH}$, the data of $\operatorname{Re}\left(J_{1}\right)$ for all three SAMs have in common that they start at negative values and increase significantly over the desorption peak (Fig. 2a, 3 and 4a). Below the desorption potential, $\operatorname{Re}\left(J_{1}\right)$ remains almost constant till the potential vertex. While for MUD and DDT, $\operatorname{Re}\left(J_{1}\right)>0$ after the desorption potential (Fig. 2a and 3), $\operatorname{Re}\left(J_{1}\right)$ remains close to 0 for ODT (Fig. 4a). For ODT, the $\operatorname{Re}\left(J_{1}\right)$ desorption curves are different compared to a capacitance measurement available in the literature, which shows an increasing capacitance after desorption..$^{23}$ The reverse anodic scans in the first cycle are clearly different between the 


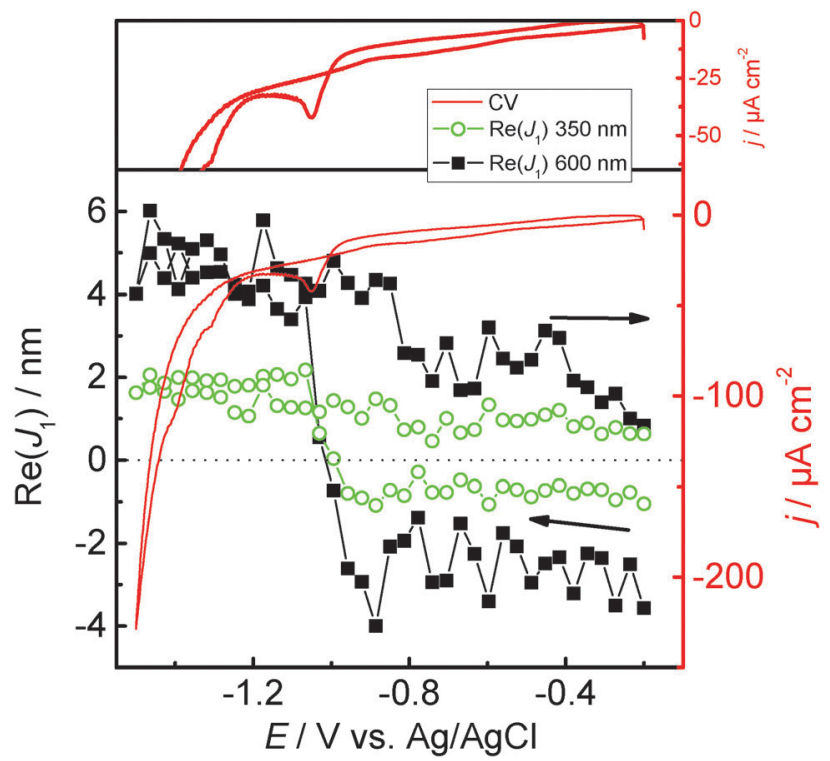

Fig. 3 First $\mathrm{CV}$ cycle $\left(-, 5 \mathrm{mV} \mathrm{s}^{-1}\right.$ ) and concurrent $\operatorname{Re}\left(\mathrm{J}_{1}\right)$ at $350 \mathrm{~nm}$ (०) and $600 \mathrm{~nm}$ ( $)$ of DDT-covered $\mathrm{Au}(111)$ in $0.1 \mathrm{M} \mathrm{NaOH}$. Arrows indicate the scan direction for the ellipsometric curves. The dotted line $(.$.$) shows$ $\operatorname{Re}\left(J_{1}\right)=0$.

different SAMs: in ODT, which shows a distinct readsorption peak in the $\mathrm{CV}, \operatorname{Re}\left(J_{1}\right)$ returns to its initial values (Fig. 4a), while for both MUD and DDT, $\operatorname{Re}\left(J_{1}\right)$ remains positive, and decreases slightly after the desorption potential (Fig. 2a and 3). The decrease stretches over more than $500 \mathrm{mV}$ in a potential range in which readsorption is expected. This behaviour can be explained with incomplete readsorption. For MUD, slight differences in the curve shapes are detected for both wavelengths (Fig. 2a).

For ODT, in the second CV cycle (Fig. 4b), both CV and ellipsometric scan appear to be very similar to the first scan, with a lower total charge in the desorption peak, but identical (in the limits of the error) ellipsometric results. However, for MUD and DDT, strong differences are observed (Fig. 2b). For the example of MUD (Fig. 2b), during the second CV cycle, the hysteresis between the $\operatorname{Re}\left(J_{1}\right)$ values becomes smaller. $\operatorname{Re}\left(J_{1}\right)$ always remains positive after first reaching a positive value. In the third scan (ESI; $\dagger$ Fig. S4), $\operatorname{Re}\left(J_{1}\right)$ still remains positive, but at even lower values. As reported for PyPP1 previously, ${ }^{42} \operatorname{Re}\left(J_{1}\right)$ becomes closer to 0 with an increasing number of scans.

Results in $0.1 \mathrm{M} \mathrm{NaClO}_{4}$ for MUD are qualitatively similar to $\mathrm{NaOH}$ (ESI; $\nmid$ Fig. S1). Comparing the CVs, it is remarkable that the current starts to increase due to HER approximately at the same potential despite the change in $\mathrm{pH}$ by 6 units. The thermodynamic start of the HER should shift here by $\approx 350 \mathrm{mV}$, which is not observed. The HER overpotential in the presence of these SAMs is therefore strongly dependent on $\mathrm{pH}$. As this SAM desorbs at the same potential in $\mathrm{NaOH}$ and $\mathrm{NaClO}_{4}$ the observation of a pH-dependent HER overpotential is not surprising, as the HER onset may be related to SAM desorption. The reductive desorption of ODT was also measured in $0.1 \mathrm{M} \mathrm{NaClO}_{4}$ and similar results were obtained (ESI $\dagger$ ). For DDT, the results in $\mathrm{NaClO}_{4}$ are qualitatively different from $\mathrm{NaOH}$ and displayed in the $\mathrm{ESI} \dagger$ (Fig. S2).
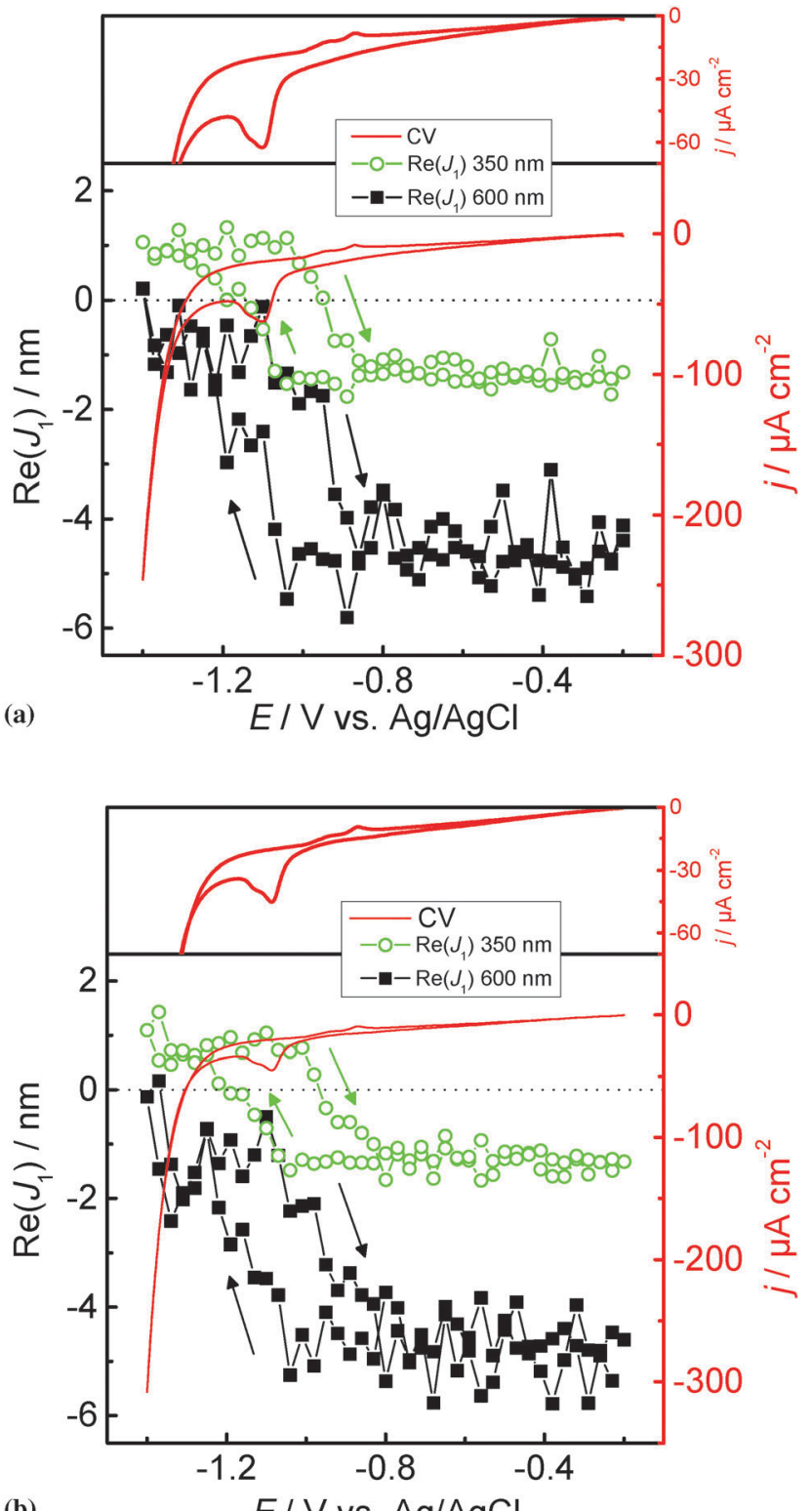

(b)

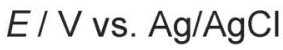

Fig. 4 (a) First and (b) second CV cycle $\left(-, 5 \mathrm{mV} \mathrm{s}^{-1}\right)$ and concurrent $\operatorname{Re}\left(J_{1}\right)$ at $350 \mathrm{~nm}(0)$ and $600 \mathrm{~nm}(\mathbf{\square})$ of ODT-covered $\mathrm{Au}(111)$ in $0.1 \mathrm{M}$ $\mathrm{NaOH}$. Arrows indicate the scan direction for the ellipsometric curves. The dotted line $(\cdots)$ shows $\operatorname{Re}\left(J_{1}\right)=0$.

Experiments in $0.1 \mathrm{M} \mathrm{HClO}_{4}$ for MUD on the other hand show a shift of the HER, so that a study of SAM-desorption is difficult in the acidic electrolyte.

Classifying the ellipsometric data recorded during the first cycle two groups emerge, which shall be termed A and B. The results are summarised in Table 1 . Type A shows a completely reversible desorption/readsorption process when scanning over the desorption potential and back. Such behaviour is recorded for ODT, and with a slight modification for DDT in $\mathrm{NaClO}_{4}$. The most common type of behaviour is type $\mathrm{B}$, a crossing of the $\operatorname{Re}\left(J_{1}\right)=0$ line during the desorption process, with $\operatorname{Re}\left(J_{1}\right)>0$ even after readsorption, i.e. a non-reversible desorption. 
Table 1 Classification of the observed ellipsometric desorption curves. The asterisk $\left(^{*}\right)$ indicates an apparent wavelength-dependence of the thickness (ESI and Table 2)

\begin{tabular}{lll}
\hline SAM & In $\mathrm{NaOH}$ & In $\mathrm{NaClO}_{4}$ \\
\hline MUD & $\mathrm{B}$ & $\mathrm{B}$ \\
DDT & $\mathrm{B}^{*}$ & $\mathrm{~A}^{*}$ \\
ODT & $\mathrm{A}^{*}$ & $\mathrm{~A}^{*}$
\end{tabular}

This desorption behaviour was previously recorded for PyPP1, ${ }^{42}$ and is found here for MUD and DDT in $\mathrm{NaOH}$.

\subsection{Interpretation of ellipsometric data}

For all intact SAMs, $\operatorname{Re}\left(J_{1}\right)<0$, in accordance with the form of $J_{1}$ for a single homogeneous layer in eqn (4), when a layer composed of a substance with a dielectric constant larger than that of the electrolyte is present on a metallic substrate. The positive values of $\operatorname{Re}\left(J_{1}\right)$ found after desorption indicate that the dielectric constant of the electrolyte in the interfacial region is (on average) smaller than the dielectric constant of the bulk electrolyte. Alternatively, the surface region of the metal may have a lower real part of dielectric constant than the bulk metal. However, strong spectral features are expected in $\operatorname{Re}\left(J_{1}\right)$ for this case, which are not observed - more details will be discussed in the second part of this section.

Following eqn (4), an increase from the initially negative $\operatorname{Re}\left(J_{1}\right)$ to 0 during the desorption process is consistent with a complete loss of the adsorbed SAM. Alternatively, the integral in the definition of $J_{1}$ [eqn (3)] shows that regions in the interface with positive and negative dielectric constant difference have opposite effects on $J_{1}$, which may cancel. The increase of $\operatorname{Re}\left(J_{1}\right)$ above 0 implies that regions with a dielectric constant below that of the solvent are generated in the desorption process. This interpretation is also consistent with the observation of $\operatorname{Re}\left(J_{1}\right)>0$ during the start of the HER (Fig. 1b). In this work, strongly positive values of $\operatorname{Re}\left(J_{1}\right)$ will be treated as a sign of the presence of $\mathrm{H}_{2}$ in the interface, as HER is thermodynamically possible at the desorption potentials. ${ }^{16}$ Because of the magnitude of the effects, the formation of a void ("layer of vacuum") between metal and desorbed SAMs is not considered here, though such a void is discussed for other systems. ${ }^{8,56-58}$ The interpretation of $\mathrm{H}_{2}$ is also consistent with previous work. ${ }^{42}$

Treating the formed $\mathrm{H}_{2}$ as a homogeneous layer, eqn (4) can be used to get an estimate of the apparent layer thickness $d_{\mathrm{H}_{2}}{ }^{\prime}$ when considering a complete loss of the SAM. Alternatively, considering the SAM to be present in a physically unmodified state after desorption, eqn (3) can be used to estimate the analogous apparent layer thickness $d_{\mathrm{H}_{2}}{ }^{*}$. In this analysis, $\varepsilon\left(\mathrm{H}_{2}\right)=1$. At each wavelength, the respective equation has been evaluated and a thickness value has been obtained as a result. Note that the wavelengthdependence of the dielectric function of the gold substrate is included in the analysis. Table 2 summarises the results, giving the minimum and maximum thickness value obtained, as well as the average, for each system. The actual effective apparent layer thickness $d$ should be between $d_{\mathrm{H}_{2}}{ }^{\prime}$ and $d_{\mathrm{H}_{2}}{ }$. Overall, the

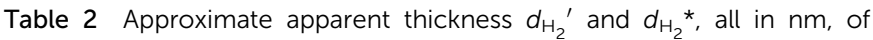
hypothetical homogeneous layers of $\mathrm{H}_{2}$ after desorption of the indicated SAM in the indicated electrolyte. For both quantities, minimum (min), maximum (max) and average $\langle d\rangle$ values are given, obtained from the analysis of a full ellipsometric spectrum from $300-820 \mathrm{~nm}$

\begin{tabular}{|c|c|c|c|c|c|c|c|}
\hline SAM & Electrolyte & $\begin{array}{l}\min \\
\left(d_{\mathrm{H}_{2}}{ }^{\prime}\right)\end{array}$ & $\left\langle d_{\mathrm{H}_{2}}{ }^{\prime}\right\rangle$ & $\begin{array}{l}\max _{\left(d_{\mathrm{H}_{2}}{ }^{\prime}\right)} \\
\end{array}$ & $\min _{\left(d_{\mathrm{H}_{2}}{ }^{*}\right)}$ & $\left\langle d_{\mathrm{H}_{2}}{ }^{*}\right\rangle$ & $\begin{array}{l}\max _{\left(d_{\mathrm{H}_{2}}{ }^{*}\right)} \\
\end{array}$ \\
\hline MUD & $\mathrm{NaOH}$ & 0.42 & $(0.51 \pm 0.06)$ & 0.70 & 0.72 & $(0.85 \pm 1.00)$ & 1.11 \\
\hline MUD & $\mathrm{NaClO}_{4}$ & 0.38 & $(0.47 \pm 0.06)$ & 0.67 & 0.70 & $(0.82 \pm 1.01)$ & 1.08 \\
\hline DDT & $\mathrm{NaOH}$ & 0.33 & $(0.7 \pm 0.2)$ & 1.25 & 0.65 & $(1.0 \pm 0.3)$ & 1.68 \\
\hline DDT & $\mathrm{NaClO}_{4}$ & 0.19 & $(0.7 \pm 0.4)$ & 1.59 & 0.48 & $(1.0 \pm 0.5)$ & 2.04 \\
\hline ODT & $\mathrm{NaOH}$ & $\mathrm{n} / \mathrm{a}$ & $\mathrm{n} / \mathrm{a}$ & 0.59 & 0.32 & $(0.7 \pm 0.3)$ & 1.44 \\
\hline ODT & $\mathrm{NaClO}_{4}$ & $\mathrm{n} / \mathrm{a}$ & $\mathrm{n} / \mathrm{a}$ & 0.53 & 0.46 & $(0.8 \pm 0.3)$ & 1.44 \\
\hline
\end{tabular}

numbers in Table 2 should be treated with caution, and should be used to illustrate the order of magnitude of the effect.

Because the thickness of the $\mathrm{H}_{2}$ layer cannot depend on the wavelength, observation of a systematic wavelength dependence hints to the presence of a phenomenon which is not included in the model presented so far. A strong wavelength dependence is, however, observed for DDT and ODT, which will be discussed below. Apparent layer thicknesses determined at the different wavelengths plotted against the wavelength (i.e. "thickness spectra") are shown in the ESI $\dagger$ (Fig. S6 and S7).

The observation of a wavelength dependence of $d$ is the consequence of a wavelength dependence of $J_{1}$ beyond the wavelength-dependence of $\varepsilon_{2}$ used here. A wavelength dependence beyond the wavelength dependence of $\varepsilon_{2}$ is also observed when following $\operatorname{Re}\left(J_{1}\right)$ in the first scan in the anodic direction after desorption of MUD (Fig. 2a), where the reverse scan after the first cathodic scan shows a decreasing $\operatorname{Re}\left(J_{1}\right)$ at $600 \mathrm{~nm}$, while $\operatorname{Re}\left(J_{1}\right)$ is constant at $350 \mathrm{~nm}$. Three possible explanations for the wavelength-dependence shall be considered, (a) changes in the absorption spectrum of the layer which is not included in the analysis, (b) a change in the gold dielectric function in the interfacial region (e.g. because of the presence of surface states, or more generically due to electroreflectance ${ }^{59,60}$ ), and (c) the optical anisotropy of the SAMs and a change in anisotropy during the desorption process.

Changes in the visible absorption spectrum of the alkyl chains can safely be excluded as the origin for any of the observed wavelength dependencies.

Differences in the dielectric function of gold between the $\mathrm{Au}(111)$ used here and the reference data used to obtain the function employed for analysis in this work are likely to be present, because of slight differences in the surface crystal structure. ${ }^{47,48}$ The electrochemical processes at the interface may lead to transitions between occupied and empty surface states, which also show as absorption features in the data of $J_{1}$ and should show distinct spectral features. ${ }^{61-65}$ Electroreflectance also shows special features in the spectra around certain characteristic wavelengths of the substrate dielectric function. ${ }^{59,66}$ Indeed spectral features are observed in the "spectra" of $d_{\mathrm{H}_{2}}{ }^{\prime}$ and $d_{\mathrm{H}_{2}}{ }^{*}$, typically at wavelengths below $550 \mathrm{~nm}$ (ESI $\dagger$ ). Fig. S5 in the ESI $\dagger$ shows, however, that at these special features, $d$ deviates only $\approx 15 \%$ from the respective $\langle d\rangle$. Therefore, the process causing this deviation can account for only $\approx 15 \%$ of the 
overall deviation observed in the ESI, $\dagger$ Fig. S6 and S7. These features can, however, account only for a deviation of $\approx 15 \%$ around the respective $\langle d\rangle$, and not for the observed systematic dependence, with minima at the highest wavelength. The large differences between minimum and maximum values by more than one order of magnitude are therefore likely not caused by such effects. Here, only the behaviour of the reverse scan of MUD in $\mathrm{NaOH}$ may be caused by a change in the interfacial electronic structure, or at least occupancy, of the gold substrate.

In particular, the majority of the observed difference cannot be caused by an interfacial change in the dielectric function due to changes in the charge state of the interface ("electroreflectance"). ${ }^{59,67}$ (Surface states can also contribute to electroreflectance spectra, ${ }^{68}$ which is not discussed here in detail.) First, the previous argument about the strong wavelength dependence holds here as well, because qualitatively different behaviour is expected in regions where $-\operatorname{Re}\left(\varepsilon_{2}\right)<1$ (e.g. around $350 \mathrm{~nm})^{53}$ and in regions where $-\operatorname{Re}\left(\varepsilon_{2}\right)>1$ (above $500 \mathrm{~nm}) .{ }^{66}$ Second, the electroreflectance effect is much smaller than the effects observed here. ${ }^{59,69}$ Electroreflectance is likely, however, to contribute to the deviations observed below $500 \mathrm{~nm}$ e.g. in the thickness spectrum of MUD (ESI $\dagger$ Fig. S5). Electroreflectance can also not account for the differences observed in the thickness spectra between MUD, DDT and ODT (ESI $\dagger$ ), because there, essentially the same behaviour would be expected for all three SAMs. To strengthen the argument, the ellipsometic data from this work may be compared to potential-dependent capacitance measurements available for ODT. ${ }^{23}$ After onset of the desorption, capacitance increases almost linearly by a factor of 5 in total, even after the desorption peak in the $\mathrm{CV} .^{23}$ If the corresponding change in surface charge and the associated change in electroreflectance were dominating the curves of $\operatorname{Re}\left(J_{1}\right), \operatorname{Re}\left(J_{1}\right)$ were expected to rise strongly after the desorption potential, which is not observed.

On the other hand, ellipsometric detection has been successfully applied to the study of optical anisotropy of organic molecules at interfaces. ${ }^{70-74}$ Electroreflectance is also affected by optical anisotropy. ${ }^{60}$ A systematic ("baseline-like") decrease or increase in the $d_{\mathrm{H}_{2}}$ "spectrum", similar to the spectrum as observed for DDT and ODT, is expected in the presence of optical anisotropy in the interfacial layer. A change in the optical anisotropy during the desorption process will lead to a systematic wavelength dependence, as will be shown below.

The simplest type of optical anisotropy possesses azimuthal symmetry: the optical axis is oriented perpendicular to the interface. Such a situation is often encountered in systems where in-plane differences average out due to domain formation, and may serve as a useful starting point for the analysis here. (A full description of an anisotropic layer is possible, ${ }^{75}$ but for systems with weak anisotropy as those investigated here the problem of data inversion is typically ill-posed.) The system can be described by a dielectric function $\varepsilon_{z}$ perpendicular to the interface and a dielectric function $\varepsilon_{x}$ parallel to the interface. ${ }^{46}$ Here, both $\varepsilon_{z}$ as well as $\varepsilon_{x}$ are treated as real, because the investigated SAMs are non-absorbing. For this situation, the definition of $J_{1}$ [eqn (3)] modifies to ${ }^{46}$

$$
J_{1}=\int_{-\infty}^{+\infty}\left[\varepsilon_{1}+\varepsilon_{2}-\frac{\varepsilon_{1} \varepsilon_{2}}{\varepsilon_{z}(z)}-\varepsilon_{x}(z)\right] \mathrm{d} z .
$$

Considering the special case of a homogeneous anisotropic layer on a metallic substrate in analogy to eqn (4) and separating real and imaginary parts yield

$$
\begin{aligned}
& \operatorname{Re}\left(J_{1}\right)=\left[\varepsilon_{1}+\operatorname{Re}\left(\varepsilon_{2}\right)-\frac{\varepsilon_{1}}{\varepsilon_{z}} \operatorname{Re}\left(\varepsilon_{2}\right)-\varepsilon_{x}\right] d, \text { and } \\
& \operatorname{Im}\left(J_{1}\right)=\left[\operatorname{Im}\left(\varepsilon_{2}\right)-\frac{\varepsilon_{1}}{\varepsilon_{z}} \operatorname{Im}\left(\varepsilon_{2}\right)\right] d .
\end{aligned}
$$

When comparing eqn (9) to (4), it is obvious that the layer dielectric constant $\varepsilon_{\mathrm{s}}$, which is present in the two final terms in eqn (4), is replaced by $\varepsilon_{x}$ in one term, and by $\varepsilon_{z}$ in the other. In the situation encountered in this work, there is a strong wavelengthdependence of $\varepsilon_{2}$, which enters one of these terms. Therefore, from the shape of both equations it is obvious that if data of an anisotropic layer are analysed with the assumption of isotropy, the resulting layer thickness $d$ will show an apparent wavelengthdependence, as observed for DDT and ODT in this work.

For a simple organic monolayer system, one could now proceed to replace $\varepsilon_{z}$ and $\varepsilon_{x}$ in eqn (9) by $\bar{\varepsilon}=\frac{1}{3} \varepsilon_{z}+\frac{2}{3} \varepsilon_{x}$ and $\Delta \varepsilon=\varepsilon_{z}-\varepsilon_{x}$. Subsequently, the system of eqn (9) could be solved for $d$ and $\Delta \varepsilon$ in analogy to the solution for isotropic systems. ${ }^{45}$ With $\bar{\varepsilon}$ from bulk measurements, this procedure yields an analytic solution to determine $d$ and $\Delta \varepsilon$. Because in this work, $J_{1}^{\text {(init) }}$ was calculated on the basis of literature data (as described in Section 2.3), and because $\bar{\varepsilon}$ cannot be obtained in an independent experiment for a system containing $\mathrm{H}_{2}$ and the remains of the organic layer, this route cannot be used for the analysis of data from this work.

Nevertheless, optical anisotropy can qualitatively explain the observed apparent wavelength-dependence in the case of DDT in $\mathrm{NaClO}_{4}$ and ODT. The main source for optical anisotropy here are the organic molecules, which have an anisotropic polarisability, and which are tilted in the intact SAM. ${ }^{11}$ Especially in the case of ODT, the completely reversible adsorption/desorption behaviour detected both in the $\mathrm{CV}$ and in the ellipsometric measurements implies that after breakage of the Au-S bond, the molecules must remain physisorbed, i.e. anisotropically ordered, near the interface. Hence, the observations are consistent with a simple model in which a planar anisotropic layer is present above the metal surface (Fig. 5a), which returns to its initial, chemisorbed state after a reversal of the electrode potential.

However, in the cases where no reversible adsorption/ desorption behaviour is observed, but the data indicate a presence of $\mathrm{H}_{2}$, different interfacial aggregates must be present. Here, we propose the presence of small bubbles of e.g. hemispherical shape, which are stabilised by the desorbed thiols, as depicted in Fig. 5b. Such structures have a large spread of orientations. Therefore, such structures appear to be almost isotropic. Such thiolate-stabilised bubbles explain the data 

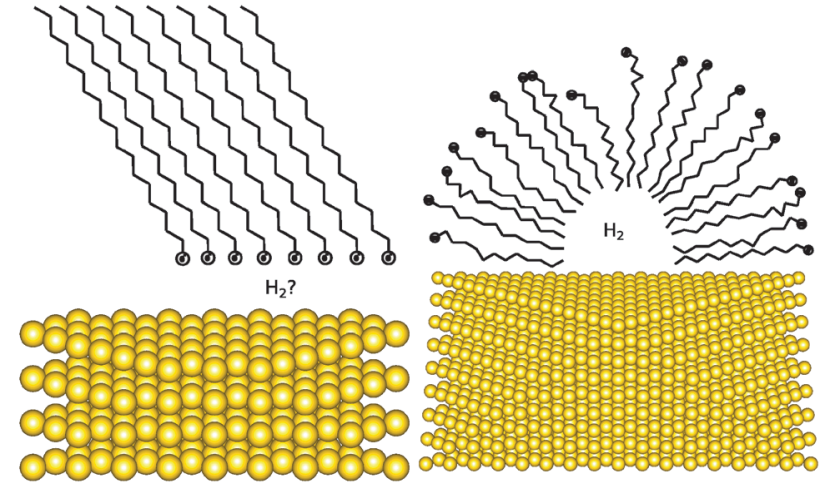

(a)

(b)

Fig. 5 Cartoon representation of the structures which may be present after breaking of the $\mathrm{Au}-\mathrm{S}$ bond. (a) Flat aggregate of SAM-forming molecules, as suggested for type $A$ (Table 1$). \mathrm{H}_{2}$ may or may not be present in the region between organic molecules and substrates. The orientation of the molecules remains similar to that in the SAM, and a reversible readsorption is possible. (b) Spherical aggregate of desorbed SAM-forming molecules, with $\mathrm{H}_{2}$ in the core. This shape is one example which may be present for desorption type B (Table 1). Molecular tails are oriented towards the hydrophobic core of the aggregate, reducing the interfacial energy between hydrogen gas and the electrolyte. A reversible readsorption of the SAM-forming molecules is impossible in this case.

obtained for MUD. For DDT in $\mathrm{NaOH}$, similar aggregates are proposed, with a remaining anisotropy, as e.g. present in cylindrical structures.

The existence of spherical aggregates after loss of SAMchemisorption has previously been established. ${ }^{30}$ As especially the alkyl SAMs are well investigated, differences in the surface structure after adsorption to explain the differences observed, in particular between DDT and ODT, can be excluded: both form a $\sqrt{3} \times \sqrt{3} \mathrm{R} 30^{\circ}$ lattice. ${ }^{11}$ Further, tilt angles of the alkyl chains of $\approx 30^{\circ}$ are commonly observed. ${ }^{11}$

The differences between the different $\mathrm{pH}$ observed for DDT are attributed to the lower hydrophilicity and hence solubility of the thiol compared to the thiolate. ${ }^{76}$ Because for $n$-alkyl thiols, $\mathrm{p} K_{\mathrm{a}} \approx 11,{ }^{76}$ a large fraction of the molecules will be present as thiols and not thiolates near neutral $\mathrm{pH}$. The low solubility of these molecules compared to the charged thiolates implies that molecules are less likely to leave the physisorbed monolayer. On the other hand, the longer-chained ODT is less soluble even as a thiolate, while hydroxylated MUD is more soluble even as a thiol. Thus, the formation of spherical aggregates on the surface can be seen as a first rearrangement step after loss of a few molecules into the solution, following the breakage of the covalent bond to the surface. This process is hypothesized to be of similar nature to the formation of a buckling instability in lipid mono- and bilayers. ${ }^{77-79}$

\section{Conclusions}

From the systems investigated here, only ODT shows a fully reversible desorption-readsorption behaviour in the ellipsometric data in both neutral and alkaline electrolytes, consistent with impedance investigations. ${ }^{18}$ DDT shows fully reversible desorption behaviour only in the neutral electrolyte. For DDT in alkaline solution and MUD, ellipsometric data can only be understood with the presence of a substance with significantly lower dielectric constant than water in the interfacial region. For ODT, the presence of such a substance cannot be excluded from the data. As desorption occurs at electrode potentials where HER is thermodynamically possible, ${ }^{16}$ this substance is likely to be $\mathrm{H}_{2}$. After initial formation of $\mathrm{H}_{2}$, it remains in the interfacial region if the electrode potential is reversed back to the oxidative readsorption region. After a few $\mathrm{CV}$ cycles over the desorption potential, the ellipsometric data for MUD and DDT approach the values expected for a complete loss of the SAM.

At near neutral $\mathrm{pH}$, ODT and MUD behave similar to that under alkaline conditions.

In $\mathrm{NaOH}$, data for MUD are consistent with models of an isotropic layer, both before as well as after desorption. On the other hand, data from DDT and ODT layers show a wavelengthdependence, which could be interpreted as a change in optical anisotropy during the desorption process. While overall the formation of spherical, micelle-like aggregates is well-established in the desorbed state, ${ }^{30}$ the role of $\mathrm{H}_{2}$ in the interfacial region is less clear. Overall, in the desorbed state, two kinds of aggregates can be discussed: on the one hand, spherical or hemispherical (Fig. 5b), and on the other hand flat, monolayer-like aggregates (Fig. 5a). Data for MUD are compatible with the formation of a spherical aggregate after desorption, where the aggregate in fact stabilises a $\mathrm{H}_{2}$ "nanobubble".** For ODT on the other hand, a flat structure dominates the desorbed state. This behaviour contrasts the observations for hexadecanethiol. ${ }^{80}$ The measurements for DDT are somewhere in between those of MUD and those of ODT. For DDT, also $\mathrm{H}_{2}$ nanobubble stabilised by desorbed molecules is consistent with the data. The results here also imply that hydrophobic, long chain thiols form SAMs which are more "stable" compared to shorter thiol SAMs: the molecules remain at the interface. ${ }^{18}$

The differences between the SAMs investigated here show that the reductive desorption process is more complex than the simple reaction equation suggests. Solubility in the electrolyte is playing a role in the loss of physisorption, with more hydrophobic (i.e. less soluble) substances tending to form more stable aggregates. More detailed information, especially in the quantification of the anisotropy, may be obtained by full Mueller matrix ellipsometry, ${ }^{81}$ provided there is sufficient sensitivity. More information on the lateral extension of the aggregate would be available from the analysis of the polarisation of scattered light. ${ }^{82,83}$

\section{Acknowledgements}

The authors thank Stefanie Pengel for help with producing the $\mathrm{Au}(111)$ substrates. Y. C. thanks for support from the European

** Control experiments show no effect of macroscopic bubbles on the ellipsometric signal, as shown in Section 3.1. Therefore, the hypothesis is that the $\mathrm{H}_{2}$ is present in a structure which at least in one dimension is significantly smaller than the wavelength of light. 
Union and the state of North Rhine-Westphalia in the frame of the HighTech NRW program. The MPIE machine shop is acknowledged for the building of the in situ SE cell. Prof. Stratmann is acknowledged for his continuous support.

\section{References}

1 J. R. T. Seddon and D. Lohse, J. Phys.: Condens. Matter, 2011, 23, 133001.

2 V. S. J. Craig, Soft Matter, 2011, 7, 40-48.

3 M. Hampton and A. Nguyen, Adv. Colloid Interface Sci., 2010, 154, 30-55.

4 X. H. Zhang, Phys. Chem. Chem. Phys., 2008, 10, 6842-6848.

5 A. Finger and D. Johannsmann, Phys. Chem. Chem. Phys., 2011, 13, 18015-18022.

6 Y.-H. Lu, C.-W. Yang and I.-S. Hwang, Langmuir, 2012, 28, 12691-12695.

7 C.-W. Yang, Y.-H. Lu and I.-S. Hwang, J. Phys.: Condens. Matter, 2013, 25, 184010.

8 Y. Takata, J.-H. J. Cho, B. M. Law and M. Aratono, Langmuir, 2006, 22, 1715-1721.

9 A. Ulman, Chem. Rev., 1996, 96, 1533-1554.

10 J. C. Love, L. A. Estroff, J. K. Kriebel, R. G. Nuzzo and G. M. Whitesides, Chem. Rev., 2005, 105, 1103-1170.

11 F. Schreiber, J. Phys.: Condens. Matter, 2004, 16, R881-R900. 12 M. Kind and C. Wöll, Prog. Surf. Sci., 2009, 84, 230-278.

13 C. Vericat, M. E. Vela, G. Benitez, P. Carro and R. C. Salvarezza, Chem. Soc. Rev., 2010, 39, 1805-1834.

14 E. Pensa, E. Cortés, G. Corthey, P. Carro, C. Vericat, M. H. Fonticelli, G. Benítez, A. A. Rubert and R. C. Salvarezza, Acc. Chem. Res., 2012, 45, 1183-1192.

15 R. Alkire, D. Kolb, J. Lipkowski and P. Ross, Chemically Modified Electrodes, Wiley-VCH, Weinheim, Germany, 2009.

16 M. Pourbaix, Atlas of electrochemical equilibria in aqueous solutions, National Association of Corrosion Engineers/Centre Belge d'Etude de la Corrosion CEBELCOR, Houston/ Bruxelles, 1974, pp. 112-121.

17 L. Srisombat, A. C. Jamison and T. R. Lee, Colloids Surf., A, 2011, 390, 1-19.

18 X. Cai and S. Baldelli, J. Phys. Chem. C, 2011, 115, 19178-19189.

19 M. Rohwerder and M. Stratmann, MRS Bull., 1999, 24, 43-47.

20 J. Mathiyarasu, S. Pathak and V. Yegnaraman, Corros. Rev., 2006, 24, 281-394.

21 F. Caprioli, F. Decker, A. G. Marrani, M. Beccari and V. D. Castro, Phys. Chem. Chem. Phys., 2010, 12, 9230-9238.

22 A. Pareek, G. N. Ankah, S. Cherevko, P. Ebbinghaus, K. J. J. Mayrhofer, A. Erbe and F. U. Renner, RSC Adv., 2013, 3, 6586-6595.

23 D. García-Raya, R. Madueño, J. M. Sevilla, M. Blázquez and T. Pineda, Electrochim. Acta, 2008, 53, 8026-8033.

24 W. Wang, S. Zhang, P. Chinwangso, R. C. Advincula and T. R. Lee, J. Phys. Chem. C, 2009, 113, 3717-3725.

25 F. Caprioli and F. Decker, J. Electroanal. Chem., 2013, 708, 68-72.
26 N. Darwish, P. K. Eggers, S. Ciampi, Y. Zhang, Y. Tong, S. Ye, M. N. Paddon-Row and J. J. Gooding, Electrochem. Commun., 2011, 13, 387-390.

27 T. Doneux, M. Steichen, A. D. Rache and C. Buess-Herman, J. Electroanal. Chem., 2010, 649, 164-170.

28 I. Thom and M. Buck, Z. Phys. Chem., 2008, 222, 739-754.

29 Y. Sato and F. Mizutani, Phys. Chem. Chem. Phys., 2004, 6, 1328-1331.

30 E. Pensa, C. Vericat, D. Grumelli, R. C. Salvarezza, S. H. Park, G. S. Longo, I. Szleifer and L. P. Méndez De Leo, Phys. Chem. Chem. Phys., 2012, 14, 12355-12367.

31 D. Hobara, K. Miyake, S.-I. Imabayashi, K. Niki and T. Kakiuchi, Langmuir, 1998, 14, 3590-3596.

32 H. Wano and K. Uosaki, Langmuir, 2001, 17, 8224-8228.

33 A. Badía, Phys. Rev. B: Condens. Matter Mater. Phys., 1999, 60, 10436-10441.

34 C. Vericat, G. Andreasen, M. E. Vela, H. Martin and R. C. Salvarezza, J. Chem. Phys., 2001, 115, 6672-6678.

35 C. Humbert, B. Busson, C. Six, A. Gayral, M. Gruselle, F. Villain and A. Tadjeddine, J. Electroanal. Chem., 2008, 621, 314-321.

36 H. Zhang and S. Baldelli, J. Phys. Chem. B, 2006, 110, 24062-24069.

37 H. Zhang, C. Romero and S. Baldelli, J. Phys. Chem. B, 2005, 109, 15520-15530.

38 Y. Zhang, Y. Tong, M. Abe, K. Uosaki, M. Osawa, Y. Sasaki and S. Ye, J. Mater. Chem., 2009, 19, 261-267.

39 P. Koelsch, M. I. Muglali, M. Rohwerder and A. Erbe, J. Opt. Soc. Am. B, 2013, 30, 219-223.

40 J. R. Casanova-Moreno and D. Bizzotto, Langmuir, 2013, 29, 2065-2074.

41 M. I. Muglali, A. Bashir, A. Terfort and M. Rohwerder, Phys. Chem. Chem. Phys., 2011, 13, 15530-15538.

42 M. I. Muglali, A. Erbe, Y. Chen, C. Barth, P. Koelsch and M. Rohwerder, Electrochim. Acta, 2013, 90, 17-26.

43 Handbook of Ellipsometry, ed. H. G. Tompkins and E. A. Irene, Springer, Heidelberg, Germany, 2005.

44 Y. Chen, P. Schneider and A. Erbe, Phys. Status Solidi A, 2012, 209, 846-853.

45 Y. Chen and A. Erbe, Surf. Sci., 2013, 607, 39-46.

46 J. Lekner, Theory of Reflection of Electromagnetic and Particle Waves, M. Nijhoff, Dordrecht, The Netherlands, 1987.

47 P. G. Etchegoin, E. C. Le Ru and M. Meyer, J. Chem. Phys., 2006, 125, 164705.

48 P. G. Etchegoin, E. C. Le Ru and M. Meyer, J. Chem. Phys., 2007, 127, 189901.

49 Handbook of Chemistry and Physics, ed. W. M. Haynes, CRC Press, Boca Raton, 94th edn, 2012.

50 C. D. Bain, E. B. Troughton, Y. T. Tao, J. Evall, G. M. Whitesides and R. G. Nuzzo, J. Am. Chem. Soc., 1989, 111, 321-335.

51 J. Liu, B. Schupbach, A. Bashir, O. Shekhah, A. Nefedov, M. Kind, A. Terfort and C. Wöll, Phys. Chem. Chem. Phys., 2010, 12, 4459-4472.

52 H. Fujiwara, Spectroscopic Ellipsometry: Principles and Applications, Wiley, Chichester, UK, 2007. 
53 R. L. Olmon, B. Slovick, T. W. Johnson, D. Shelton, S.-H. Oh, G. D. Boreman and M. B. Raschke, Phys. Rev. B: Condens. Matter Mater. Phys., 2012, 86, 235147.

54 S.-S. Wong and M. D. Porter, J. Electroanal. Chem., 2000, 485, 135-143.

55 C.-J. Zhong and M. D. Porter, J. Electroanal. Chem., 1997, 425, 147-153.

56 A. Stocco and K. Tauer, Eur. Phys. J. E: Soft Matter Biol. Phys., 2009, 30, 431-438.

57 T. R. Jensen, M. Østergaard Jensen, N. Reitzel, K. Balashev, G. H. Peters, K. Kjaer and T. Bjørnholm, Phys. Rev. Lett., 2003, 90, 086101.

58 U. K. Sur and V. Lakshminarayanan, J. Colloid Interface Sci., 2002, 254, 410-413.

59 A. Brodsky, L. Daikhin and M. Urbakh, J. Electroanal. Chem. Interfacial Electrochem., 1984, 171, 1-52.

60 T. Sagara, in Advances in Electrochemical Science and Engineering: Diffraction and Spectroscopic Methods in Electrochemistry, ed. R. C. Alkire, D. M. Kolb, J. Lipkowski and P. N. Ross, Wiley-VCH, Weinheim, Germany, UV-Visible Reflectance Spectroscopy of Thin Organic Films at Electrode Surfaces, 2008, ch. 2, vol. 9, pp. 47-95.

61 S. Liu, C. Hinnen, C. N. V. Huong, N. de Tacconi and K. Ho, J. Electroanal. Chem. Interfacial Electrochem., 1984, 176, 325-338.

62 C. V. Huong, J. Electroanal. Chem. Interfacial Electrochem., 1986, 207, 235-246.

63 P. Kowalczyk, M. Puchalski, W. Kozłowski, P. Dbrowski, Z. Klusek and W. Olejniczak, Appl. Surf. Sci., 2008, 254, 4572-4576.

64 T. Brandt, M. Hövel, B. Gompf and M. Dressel, Phys. Rev. B: Condens. Matter Mater. Phys., 2008, 78, 205409.
65 P. Mishra, T. Uchihashi and T. Nakayama, Appl. Phys. Lett., 2012, 100, 141609.

66 K. Kempa, Surf. Sci., 1985, 157, L323-L327.

67 J. McIntyre, Surf. Sci., 1973, 37, 658-682.

68 F. Henglein, D. Kolb, L. Stolberg and J. Lipkowski, Surf. Sci., 1993, 291, 325-336.

69 F. Chao and M. Costa, Surf. Sci., 1983, 135, 497-520.

70 M. Thoma, M. Schwendler, H. Baltes, C. A. Helm, T. Pfohl, H. Riegler and H. Möhwald, Langmuir, 1996, 12, 1722-1728.

71 R. Sigel and G. Strobl, J. Chem. Phys., 2000, 112, 1029-1039. 72 Z. Salamon and G. Tollin, Biophys. J., 2001, 80, 1557-1567.

73 A. Erbe and R. Sigel, Eur. Phys. J. E: Soft Matter Biol. Phys., 2007, 22, 303-309.

74 D. Den Engelsen, Surf. Sci., 1976, 56, 272-280.

75 M. Schubert, Phys. Rev. B: Condens. Matter Mater. Phys., 1996, 53, 4265-4274.

76 D. L. Yabroff, Ind. Eng. Chem., 1940, 32, 257-262.

77 A. Saint-Jalmes and F. Gallet, Eur. Phys. J. B, 1998, 2, 489-494.

78 L. Courbin, J. P. Delville, J. Rouch and P. Panizza, Phys. Rev. Lett., 2002, 89, 148305.

79 H. Vandeparre and P. Damman, Phys. Rev. Lett., 2008, 101, 124301.

80 M. Byloos, H. Al-Maznai and M. Morin, J. Phys. Chem. B, 1999, 103, 6554-6561.

81 E. Garcia-Caurel, A. De Martino, J.-P. Gaston and L. Yan, Appl. Spectrosc., 2013, 67, 1-21.

82 A. Erbe, K. Tauer and R. Sigel, Langmuir, 2009, 25, 2703-2710.

83 R. Sigel, Curr. Opin. Colloid Interface Sci., 2009, 14, 426-437. 